\title{
Facile Synthesis of Copper Oxide Nanoparticles via Electrospinning
}

\author{
Abdullah Khalil, ${ }^{1}$ Mustapha Jouiad, ${ }^{1}$ Marwan Khraisheh, ${ }^{2}$ and Raed Hashaikeh ${ }^{1}$ \\ ${ }^{1}$ Department of Mechanical and Materials Engineering, Masdar Institute of Science and Technology, P.O. Box 54224, Abu Dhabi, UAE \\ ${ }^{2}$ Qatar Environment and Energy Research Institute, Qatar Foundation, Doha, Qatar \\ Correspondence should be addressed to Raed Hashaikeh; rhashaikeh@masdar.ac.ae
}

Received 13 March 2014; Accepted 16 June 2014; Published 22 July 2014

Academic Editor: Shijun Liao

Copyright (C) 2014 Abdullah Khalil et al. This is an open access article distributed under the Creative Commons Attribution License, which permits unrestricted use, distribution, and reproduction in any medium, provided the original work is properly cited.

\begin{abstract}
A novel approach for synthesizing copper oxide $(\mathrm{CuO})$ nanoparticles (NPs) through electrospinning is reported. The approach is based on producing rough and discontinuous electrospun nanofibers from a precursor based on copper acetate salt and polyvinyl alcohol (PVA) polymer. Selectively removing the polymeric phase from the fibers produced highly rough $\mathrm{CuO}$ nanofibers, which were composed of NPs that are weakly held together in a one-dimensional (1D) manner. Sonication in a suitable liquid under controlled conditions completely disintegrated the nanofibers into NPs, resulting in the formation of uniform CuO NPs suspension. Aberration corrected high resolution transmission electron microscope (HRTEM) showed that the obtained NPs are highly crystalline and nearly sphere-like with a diameter of 30 to $70 \mathrm{~nm}$. Thus, electrospinning, which is a low cost and industrially scalable technique, can also be employed for economic and large scale synthesis of NPs.
\end{abstract}

\section{Introduction}

Electrospinning, which came to light more than seven decades ago [1], turned out to be such a powerful and versatile technique for continuous and large scale production of nanofibers. The process involves the application of strong electric field to generate electrically charged jet from the viscous solution through a tiny nozzle. As the electrical potential overcomes the surface tension of the solution droplet coming out of the nozzle, the jet emerges from the droplet end and continues to thin as it approaches the collector. The solvents evaporate from the jet to form fibers having diameter of the orders of nanometer which are collected on a grounded electrode. The process has been extensively employed for fabricating a variety of polymeric nanofibers [2]. Also, by varying the electrospinning configuration and environmental parameters, fibers with varying morphology such as porous and core-shell [3] can also be produced. Moreover, by adding a certain salt in specific proportion to the polymeric precursor, nanofibers of various ceramics can also be obtained after selectively removing the polymeric phase through heat treatment [4-6].
Recently, electrospinning has also been employed for producing metallic nanowires following the same approach $[7,8]$. The approach is similar except one additional step of reduction to transform the metal oxide nanofibers into pure metal nanowires.

Whereas the use of electrospinning process is limited to the production of mats made out of nanofibers to date, we have found this process to be capable of producing nanoparticles (NPs) as well. We demonstrate this approach through an example of copper (II) oxide (CuO) NPs-a narrow band gap semiconducting material having potential catalytic [9], sensing [10], antimicrobial [11, 12], heat transfer $[13,14]$, and photovoltaic [15] applications because of their high surface to volume ratio as compared to bulk counterpart and quantum confinement effects [16].

It is worth noticing that several physical methods such as laser ablation [17] and microwave irradiation [18] and chemical techniques such as alcohothermal method $[19,20]$ and fast precipitation [21, 22] have been applied for synthesizing $\mathrm{CuO}$ NPs. However, these processes are costly in terms of energy consumption and setup requirements and hence not feasible for economic mass production at the industrial scale. 
In addition, other techniques such as "supercritical water hydrothermal synthesis" have been reported as more versatile approaches for producing NPs at the industrial scale and in a continuous manner [23]. Indeed, large variety of ceramic NPs in various size ranges have been synthesized in bulk quantities using this technique [24, 25].

In contrast, we report here electrospinning, which is an economic and industrially scalable technique [26], as an alternative and novel route for quick, low cost, and large scale production of NPs. We demonstrate this concept with the example of electrospun $\mathrm{CuO}$ nanofibers transforming to $\mathrm{CuO}$ NPs. Similar approach can be adopted for synthesizing a variety of other NPs based on the appropriate precursor composition used for electrospinning.

\section{Materials and Methods}

The viscous and uniform precursor, based on copper (II) acetate (Sigma-Aldrich) and polyvinyl alcohol (PVA) $\left(M_{w}=\right.$ 61000 , Sigma-Aldrich), was electrospun at a flow rate of $0.2 \mathrm{~mL} / \mathrm{hr}$ and a voltage of $29 \mathrm{kV}$. The details of precursor preparation, the optimized electrospinning parameters and the post-treatment conditions of fibers were based on our previous investigation [27]. In a typical experiment for converting the nanofibers to NPs, around $25 \mathrm{mg}$ of calcined nanofibers was transferred to a beaker containing $50 \mathrm{~mL}$ of water. This mixture was then ultrasonicated using probe sonication for $15 \mathrm{~min}$. This step resulted in the formation of transparent NPs suspension of $\mathrm{CuO}$ in water. The morphology of NPs was analyzed through scanning electron microscope (SEM) and their compositional analysis was carried out using energy dispersive spectroscopy (EDS) and $\mathrm{X}$-ray diffraction (XRD). The NPs were also characterized in terms of size and crystallinity using aberration corrected HRTEM. The average size of NPs was also confirmed through particle size analyzer based on the principle of "dynamic light scattering" (DLS).

\section{Results}

3.1. Morphology of As-Spun Fibers. It is well admitted that in electrospinning, the solution flow rate and the voltage are the most important processing parameters that have the greatest influence over the obtained nanofibers morphology and features. Therefore, optimum values of these two parameters must be selected for stable "Taylor cone" at the needle end and for the formation of smooth and continuous nanofibers $[28,29]$. For every solution, there is an optimum set of flow rates and voltages depending upon its viscosity and conductivity that will yield the nanofibers with maximum uniformity and continuity. Since the flow rate is related to the solution delivery to the needle and the voltage is related to taking the solution away from the Taylor cone, any imbalance between these two parameters will cause the hydrodynamic instabilities that will lead to the formation of discontinuous and nonuniform fibers with irregular morphologies [30]. In case the as-spun fibers are composite comprising both the polymer and the salt, the fiber morphology after

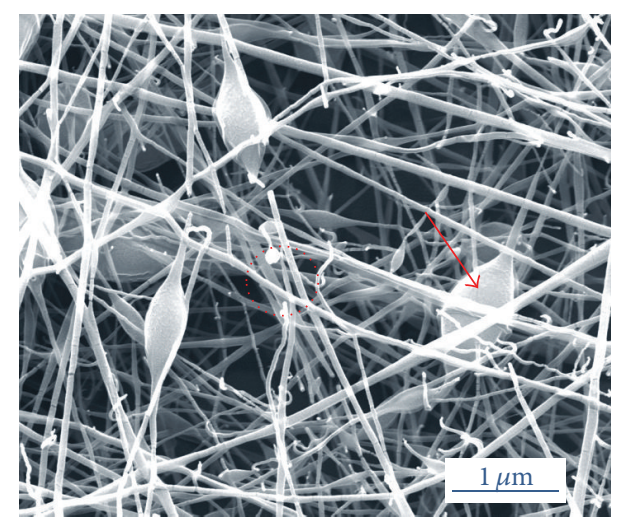

FIGURE 1: SEM image of as-spun composite nanofibers with highly defective morphology. Broken junctions and beads are marked with dotted circle and arrow, respectively.

polymer removal will also be affected by the solution flow rate and the voltage employed during the electrospinning. If the values of these two parameters are such that poor balance is maintained between solution delivery and solution removal from the needle end, not only the morphology of as-spun composite fibers becomes highly defective, but also the morphology of calcined nanofibers becomes very defective and nonuniform. In our case, $0.2 \mathrm{~mL} / \mathrm{hr}-20 \mathrm{kV}$ and $0.5 \mathrm{~mL} / \mathrm{hr}-29 \mathrm{kV}$ were found to be the optimized sets of flow rates and voltages for producing uniform and continuous nanofibers. If one of the parameters in these sets is interchanged, the result is highly discontinuous and nonuniform fibers due to imbalance between electrostatic and viscoelastic forces. Figure 1 shows the SEM image of as-spun composite nanofibers obtained at $0.2 \mathrm{~mL} / \mathrm{hr}-29 \mathrm{kV}$. The nanofibers are characterized by pronounced breaking and beading which were expected due to unstable jet formation during electrospinning. Similar morphology was observed for $0.5 \mathrm{~mL} / \mathrm{hr}-$ $20 \mathrm{kV}$.

3.2. Morphology of Fibers after Polymer Removal. Figure 2(a) shows the SEM images of calcined nanofibers. An important observation is the curling and spiraling behavior of these calcined nanofibers. As evident from Figure 1, the as-spun composite fibers are fairly straight but such quality of straightness is not evident in calcined nanofibers. The observed spiraling of calcined nanofibers is due to the residual strain which was built up when the different crystalline domains (or NPs) of $\mathrm{CuO}$ merge together to form linear 1D structure while the PVA decomposes. Figures 2(b) and 2(c) show the high magnification SEM images of calcined nanofibers where it can be clearly seen that the nanofibers are made up of NPs attached together in a linear fashion to give the nanofiber shape. It is important to mention that the electrospinning voltage and flow rate are not solely responsible for this extremely rough fiber morphology. An equally critical parameter is the precursor composition. For obtaining such morphology, it is vital to prepare a precursor which is heavily loaded with salt. As stated in the experimental section, the precursor has a polymer to salt weight ratio of $1.5: 1$ with 


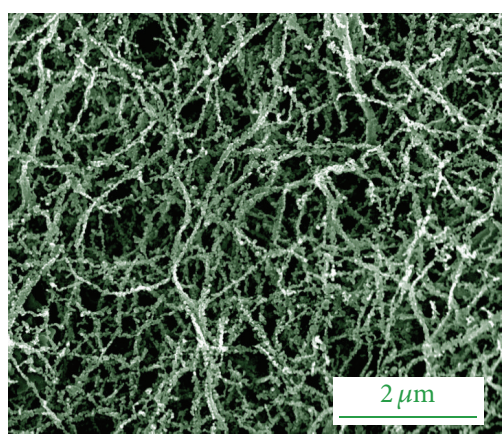

(a)

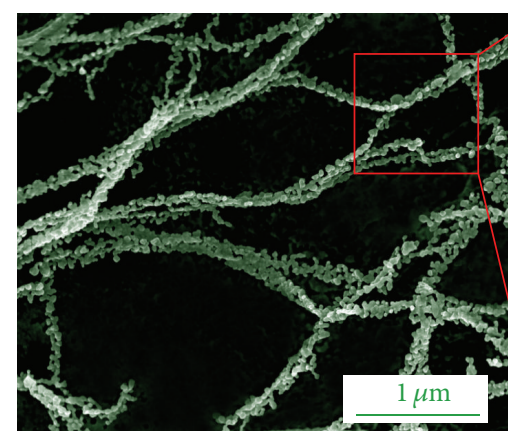

(b)

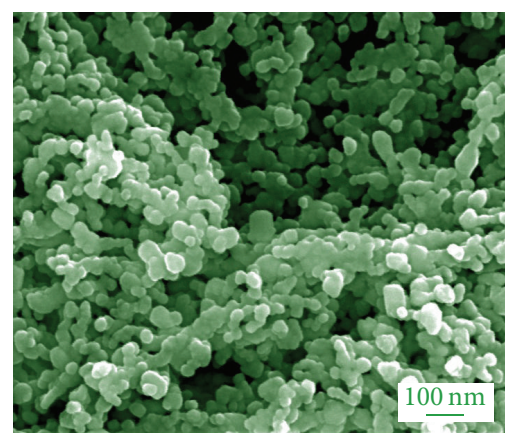

(d)

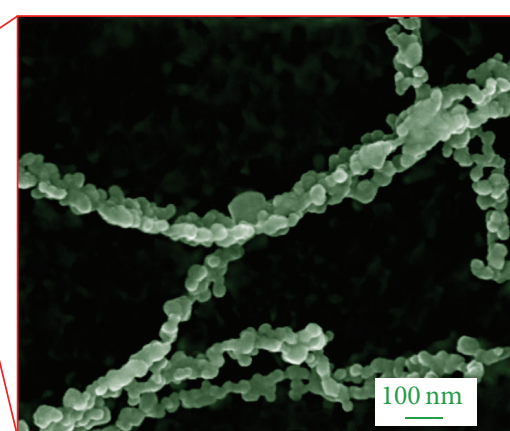

(c)

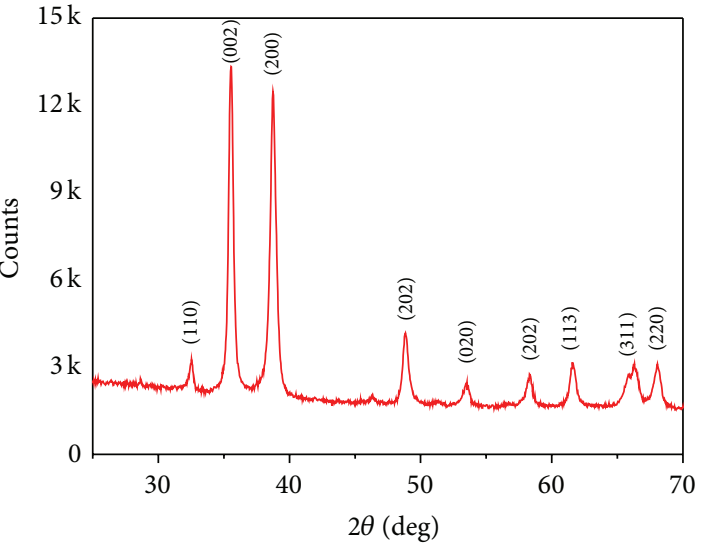

(e)

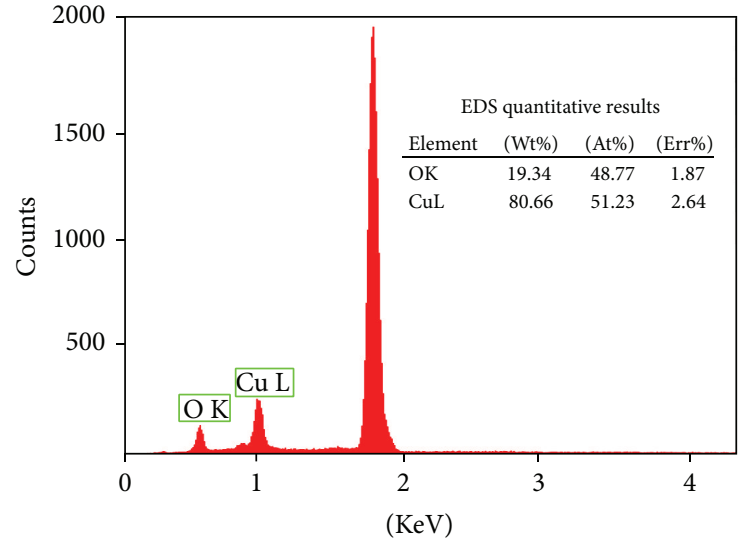

(f)

Figure 2: (a) SEM image of CuO nanofibers obtained after calcination. ((b) and (c)) Magnified images of (a). (d) Nanofibers changing to NPs' clusters after sonication in water. (e) XRD spectrum of CuO NPs. (f) EDS spectrum and quantitative results of CuO NPs (the longest peak in EDS spectrum corresponds to $\mathrm{Si}$, which was used as a substrate for collecting nanofibers and its contribution was excluded from the quantitative results).

only $1 \mathrm{~mL}$ additional water. The available water from the polymer solution and this separately added $1 \mathrm{~mL}$ water are far below the minimum amount required for forming clear and saturated $\mathrm{CuAc}$ aqueous solution. Thus, the precursor formed is basically a sol-gel comprised of CuAc particles uniformly distributed across the solution. Such high loading of salt causes dense confinement of salt across the nanofiber which ultimately results in the formation of $\mathrm{CuO}$ NPs connected together in linear $1 \mathrm{D}$ pattern. The XRD spectrum with indexed peaks, shown in Figure 2(e), confirms the purity and crystallinity of $\mathrm{CuO}$ NPs [31]. The EDS spectra and the quantitative results for NPs, shown in Figure 2(f), match well with the stoichiometric ratio of $\mathrm{Cu}$ and $\mathrm{O}$ in pure $\mathrm{CuO}$, conforming to the fact that the obtained NPs are nearly pure $\mathrm{CuO}$.

3.3. HRTEM Analysis of $\mathrm{CuO}$ NPs. The fabricated CuO NPs were examined using HRTEM. The samples were prepared by putting a tiny droplet of freshly sonicated water based $\mathrm{CuO} \mathrm{NP}$ suspension on a carbon supported $300 \mathrm{~nm}$ mesh TEM copper grid. The typical CuO NPs structure is given in Figure 3(a); the NPs appear to have a spherical-like shape with an average size of 20 to $40 \mathrm{~nm}$. They seem also to be locally clustered which may be attributed to very low evaporation rate of water used as solvent leading to particles aggregation. At higher magnification (Figure 3(b)) all 


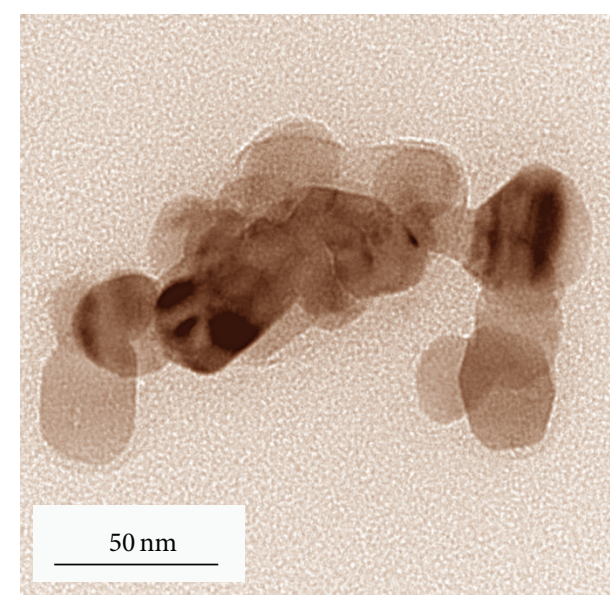

(a)

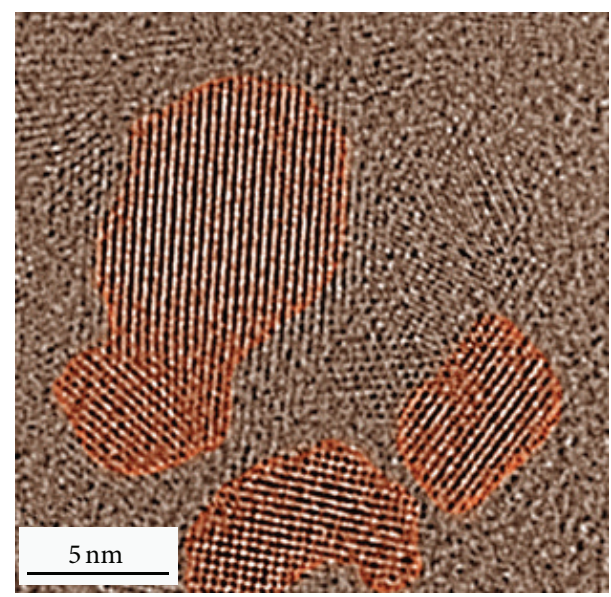

(b)

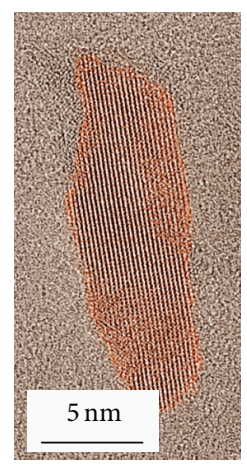

(c)

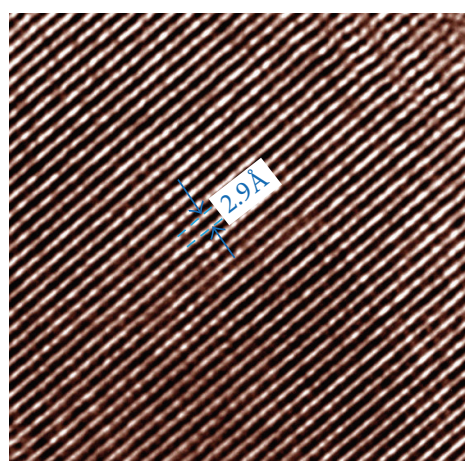

(d)

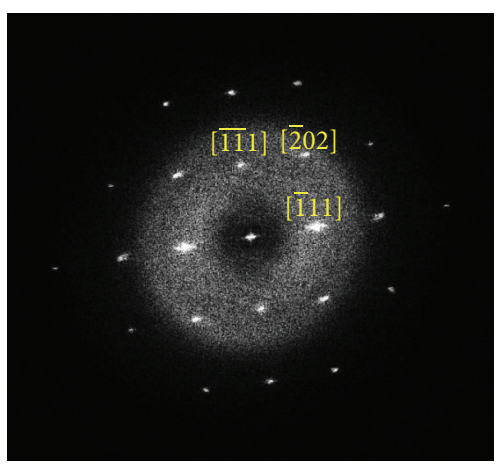

(e)

FIGURE 3: (a) TEM image of clustered CuO NPs. (b) HRTEM image of CuO NP showing high degree of crystallinity with random orientations. (c) HRTEM examination of single CuO NP. (d) Zoom-in view of crystal showing well aligned atomic planes with $2.9 \AA$ spacing. (e) FFT of the image shown in (c) matching the [101] zone axis for the monoclinic structure.

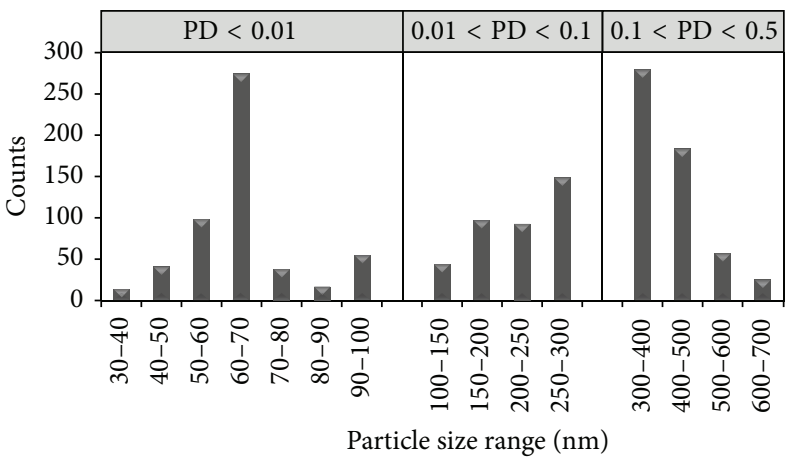

(a)

\begin{tabular}{cccc}
\hline Run number & Size $(\mathrm{nm})$ & Polydispersity & Zeta potential $(\mathrm{mV})$ \\
\hline 1 & 39.8 & 0.022 & -10.91 \\
2 & 30.5 & 0.005 & -13.88 \\
3 & 50.1 & 0.073 & -19.45 \\
4 & 240.5 & 0.169 & -20.99 \\
5 & 323.5 & 0.194 & -24.43 \\
6 & 460.9 & 0.273 & -18.35 \\
7 & 514.4 & 0.299 & -25.03 \\
8 & 571.9 & 0.317 & -21.98 \\
9 & 529.2 & 0.311 & -29.34 \\
10 & 657.4 & 0.412 & -26.38 \\
\hline
\end{tabular}

(b)

FIgure 4: (a) CuO NPs size distribution and (b) typical example of a test consisting of 10 runs conducted for estimating CuO NP size.

observed $\mathrm{CuO}$ NPs exhibit atomic fringes with a specific orientation. This is clear evidence that the synthetized $\mathrm{CuO}$ NPs are all single crystals with random orientations. Further HRTEM examination of single $\mathrm{CuO} \mathrm{NP}$ is presented in Figure 3(c): this NP structure was highlighted through (101) zone axis given in fast Fourier transformed (FFT) image in Figure 3(e). The atomic columns of this nanocrystal seem to be lying along $\left[\begin{array}{lll}\overline{1} & \overline{1} & 1\end{array}\right]$ direction. The zoom in view of this NP structure is given in Figure 3(d) which allows measuring the interplanar spacing of $d=2.9 \AA$. Further analysis of the FFT data allows determining the lattice parameters of this nanocrystal through the indexation of all visible spots and the angles between different orientations. Our measurement gives these values $(a=4.12 \AA)$ and $\left(\alpha=\gamma=89^{\circ} ; \beta=100^{\circ}\right)$. 
This result is in good agreement with the crystal structure of monoclinic $\mathrm{CuO}$ as given in the literature $(a=4.6837 \AA$, $b=3.4226 \AA$, and $c=5.1288 \AA)$ and $\left(\alpha=\gamma=90^{\circ}\right.$, $\beta=99.54^{\circ}$ ).

3.4. Particle Size Analysis through DLS. Figure 4(a) gives the DLS size distribution of $\mathrm{CuO}$ particles in freshly sonicated water suspension. It seems that the $\mathrm{CuO}$ NPs exhibit a scatter size distribution varying between 30 and $700 \mathrm{~nm}$. However, the analysis of the polydispersity (PD) and zetapotential (ZP) values must be undertaken carefully to draw a reasonable conclusion about average NP size and suspension stability. For the particles ranging between 30 and $100 \mathrm{~nm}$, the corresponding PD was found to be below 0.01 . This shows that the suspension is monodisperse and the average particle size lies below $100 \mathrm{~nm}$. Maximum counts were recorded for the size range between 60 and $70 \mathrm{~nm}$ representing that the majority of NPs fall within this size range. For higher particle size, the corresponding PD values were also higher representing NP clustering. Lower particle size and PD were observed for the initial runs and both of these values kept on increasing for the latter runs as it can be seen from a typical test result presented in Figure 4(b). This gives strong indication that the $\mathrm{CuO}$ NPs suspension is highly instable due to coagulation of NPs which happens within few minutes. Also, the ZP measurements for each run listed in the last column of Figure 3 (b) confirm the suspension instability. The average of ten $\mathrm{ZP}$ readings shown is around $21 \mathrm{mV}(<30 \mathrm{mV})$ confirming suspension instability [32]. Also, the increasing trend in the ZP with number of runs confirms that the NP coagulation increases with time. We found this clustering of NPs to be reversible; that is, if sonicated again, the suspension displayed smaller particle size with lower PD and ZP like initial runs. Based on these observations, it can be said with confidence that the average size for the majority of individual NPs is below $100 \mathrm{~nm}$ with most of them lying typically in the range 60 to $70 \mathrm{~nm}$ which is in good agreement with the size estimated from TEM images.

\section{Discussion}

Our results show that using an appropriate precursor and an optimized set of electrospinning parameters, very rough nanofibers resembling $1 \mathrm{D}$ arrays of NPs can be generated. If sonicated under controlled conditions in a liquid medium (such as water), these NPs can be easily separated resulting in the formation of NPs suspension. The NPs are usually stored and applied in the form of suspensions made with the suitable liquid. Thus, the necessary step for converting the nanofiber meshes to NPs is putting them in liquid and then performing controlled sonication leading to homogenous NP suspension.

The highly crystalline nature of $\mathrm{CuO}$ NPs reflects another potential benefit of the present approach. Despite several instabilities involved in the electrospinning, the obtained NPs were found to be nearly single crystal. The role of intermediate processing steps, that is, the polymer removal and the sonication, can be significant in this regard. The heat treatment of nanofibers at $475^{\circ} \mathrm{C}$ with low heating and cooling rates can cause the individual NPs inside the nanofiber to crystallize. Similarly, the sonication step can also lead to some recrystallization and spherical shape formation of NPs as they get an opportunity to minimize their energy and surface area.

The key benefit of using electrospinning technique for producing NPs mainly lies in its economic, facile, and industrially scalable nature. Further investigations and optimization can make this technique become an economic choice for producing NPs at the upscaling level with greater yields. Another attraction of this approach is its versatility; that is, the NPs of any ceramic material such as $\mathrm{ZnO}, \mathrm{NiO}$, $\mathrm{TiO}$, and $\mathrm{FeO}$ can be fabricated through proper choice of salt in the precursor preparation.

An indirect approach has been demonstrated for producing metal NPs via electrospinning [33]; however, the method is totally different as compared to our approach. It involves electrospinning the solution of polyacrylonitrile, acrylic acid, and $\mathrm{PdCl}_{2}$ and then reducing the as-spun nanofiber mats with hydrazine which results in the formation of Pd NPs uniformly distributed over the nanofiber mat. Because of the very low yield and adhesion of NPs to the polymeric nanofibers, the approach seems difficult to achieve a mass production level. An attempt to fabricate NPs in a continuous manner, employing the concept of bubble electrospinning, has also been demonstrated; however, the obtained product was in the form of huge clusters ( 5 microns approximately) comprising irregularly shaped particles with an average size of several hundred nanometers [34]. However our work shows that, besides producing nanofibers, electrospinning is also a promising and straightforward technique for producing well dispersed and less than $100 \mathrm{~nm} \mathrm{CuO} \mathrm{NPs} \mathrm{suspension}$ in bulk quantities and we intend to extend this work for other ceramic NPs in future.

\section{Conclusion}

A new process to synthesize copper oxide nanoparticles (NPs) in bulk quantities via electrospinning has been developed. The complete process is schematically summarized and illustrated in Figure 5. The key to properly implement this process for making $\mathrm{CuO}$ NPs is the formation of suitable precursor consisting of an appropriate salt and polymer and selection of optimized electrospinning parameters so that irregular and discontinuous 1D arrays of NPs are generated instead of regular and continuous fibers. These NP arrays are then broken into isolated NPs by sonication in appropriate liquid resulting in NPs suspension. By altering the NP concentration or adding suitable surfactant, the coagulation of these NPs can be significantly reduced. The produced particles are crystalline and have a relatively narrow particle size distribution. Moreover, this study showed the interesting potential of electrospinning technique for producing NPs. Hence, further optimization using different precursor compositions and electrospinning parameters will lead to controlled quality and mass production of NPs. 


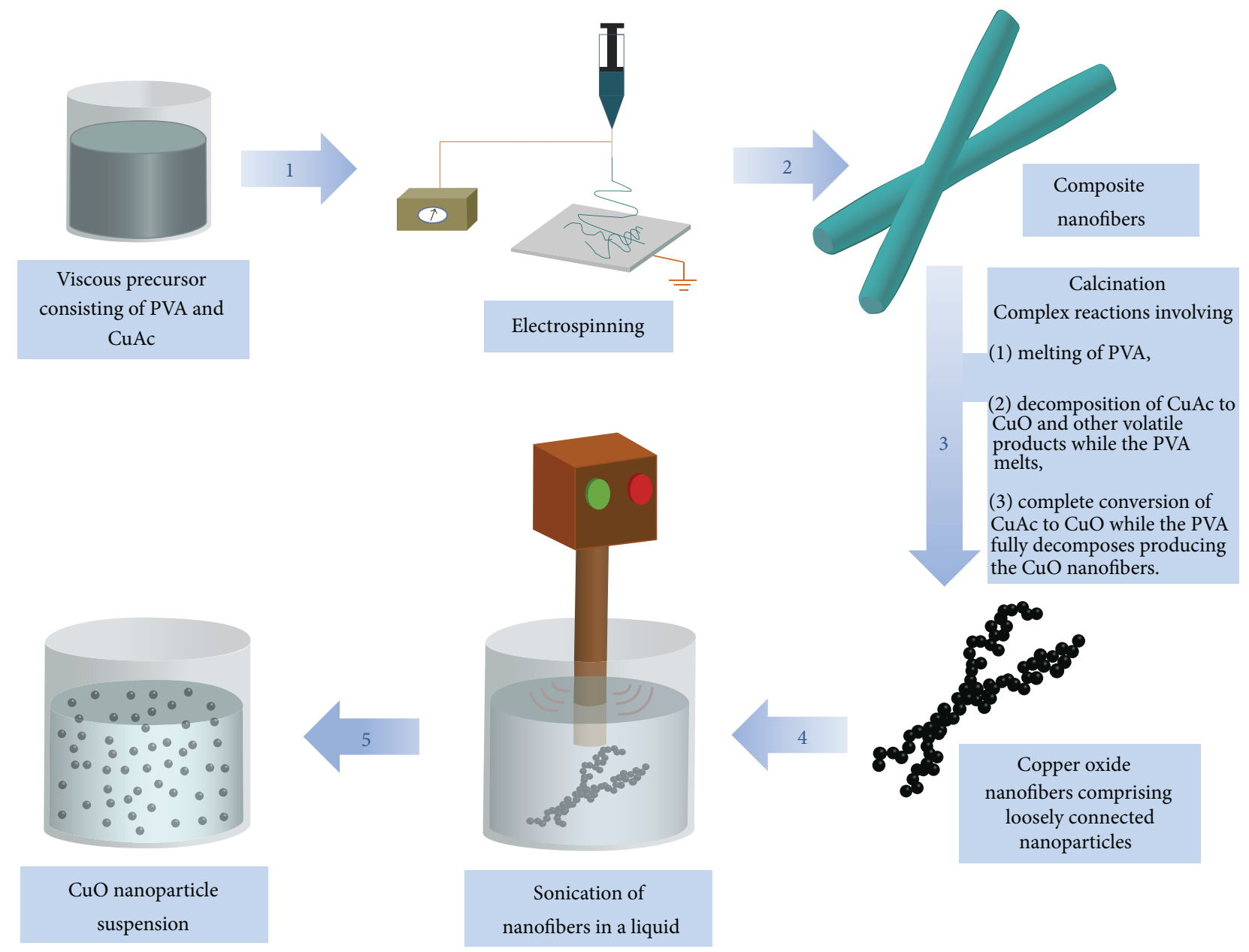

FIgURE 5: Process flow for making CuO NPs via electrospinning technique.

\section{Conflict of Interests}

The authors declare that there is no conflict of interests regarding the publication of this paper.

\section{References}

[1] A. Formhals, “US Patent 1975504," 1934.

[2] T. Subbiah, G. S. Bhat, R. W. Tock, S. Parameswaran, and S. S. Ramkumar, "Electrospinning of nanofibers," Journal of Applied Polymer Science, vol. 96, no. 2, pp. 557-569, 2005.

[3] J. T. McCann, D. Li, and Y. Xia, "Electrospinning of nanofibers with core-sheath, hollow, or porous structures," Journal of Materials Chemistry, vol. 15, no. 7, pp. 735-738, 2005.

[4] G. Larsen, R. Velarde-Ortiz, K. Minchow, A. Barrero, and I. G. Loscertales, "A method for making inorganic and hybrid (organic/inorganic) fibers and vesicles with diameters in the submicrometer and micrometer range via sol-gel chemistry and electrically forced liquid jets," Journal of the American Chemical Society, vol. 125, no. 5, pp. 1154-1155, 2003.

[5] S. S. Choi, S. G. Lee, S. S. Im, S. H. Kim, and Y. L. Joo, "Silica nanofibers from electrospinning/sol-gel process," Journal of Materials Science Letters, vol. 22, no. 12, pp. 891-893, 2003.
[6] Y. Wang and J. J. Santiago-Avilés, "Synthesis of lead zirconate titanate nanofibres and the Fourier-transform infrared characterization of their metallo-organic decomposition process," Nanotechnology, vol. 15, no. 1, pp. 32-36, 2004.

[7] H. Wu, L. Hu, M. W. Rowell et al., "Electrospun metal nanofiber webs as high-performance transparent electrode," Nano Letters, vol. 10, no. 10, pp. 4242-4248, 2010.

[8] N. S. Hansen, D. Cho, and Y. L. Joo, "Metal nanofibers with highly tunable electrical and magnetic properties via highly loaded water-based electrospinning," Small, vol. 8, no. 10, pp. 1510-1514, 2012.

[9] G. Molteni, C. L. Bianchi, G. Marinoni, N. Santo, and A. Ponti, " $\mathrm{Cu} / \mathrm{Cu}$-oxide nanoparticles as catalyst in the "click" azidealkyne cycloaddition," New Journal of Chemistry, vol. 30, no. 8, pp. 1137-1139, 2006.

[10] C. Batchelor-McAuley, G. G. Wildgoose, R. G. Compton, L. Shao, and M. L. H. Green, "Copper oxide nanoparticle impurities are responsible for the electroanalytical detection of glucose seen using multiwalled carbon nanotubes," Sensors and Actuators B: Chemical, vol. 132, no. 1, pp. 356-360, 2008.

[11] S. Jadhav, S. Gaikwad, M. Nimse, and A. Rajbhoj, "Copper oxide nanoparticles: synthesis, characterization and their antibacterial activity," Journal of Cluster Science, vol. 22, no. 2, pp. 121-129, 2011. 
[12] G. Ren, D. Hu, E. W. C. Cheng, M. A. Vargas-Reus, P. Reip, and R. P. Allaker, "Characterisation of copper oxide nanoparticles for antimicrobial applications," International Journal of Antimicrobial Agents, vol. 33, no. 6, pp. 587-590, 2009.

[13] M. H. Chang, H. S. Liu, and C. Y. Tai, "Preparation of copper oxide nanoparticles and its application in nanofluid," Powder Technology, vol. 207, no. 1-3, pp. 378-386, 2011.

[14] S. Asthana, S. Rattan, and M. Das, "Comparative studies of copper oxide with aluminium oxide nanoparticles in conventional thermal fluids for its enhanced efficiency as coolant," Proceedings of the National Academy of Sciences, India A: Physical Sciences, vol. 83, no. 2, pp. 73-77, 2013.

[15] H. Kidowaki, T. Oku, T. Akiyama, A. Suzuki, B. Jeyadevan, and J. Cuya, "Fabrication and characterization of CuO-based solar cells," Journal of Materials Science Research, vol. 1, no. 1, pp. 138143, 2012.

[16] K. Borgohain, J. B. Singh, M. V. R. Rao, T. Shripathi, and S. Mahamuni, "Quantum size effects in $\mathrm{CuO}$ nanoparticles," Physical Review B, vol. 61, no. 16, pp. 11093-11096, 2000.

[17] K. Suzuki, N. Tanaka, A. Ando, and H. Takagi, "Size-selected copper oxide nanoparticles synthesized by laser ablation," Journal of Nanoparticle Research, vol. 14, no. 5, article 0863, 2012.

[18] H. Wang, J. Z. Xu, J. J. Zhu, and H. Y. Chen, "Preparation of $\mathrm{CuO}$ nanoparticles by microwave irradiation," Journal of Crystal Growth, vol. 244, no. 1, pp. 88-94, 2002.

[19] Z. Hong, Y. Cao, and J. Deng, "A convenient alcohothermal approach for low temperature synthesis of $\mathrm{CuO}$ nanoparticles," Materials Letters, vol. 52, no. 1-2, pp. 34-38, 2002.

[20] T. Kida, T. Oka, M. Nagano, Y. Ishiwata, and X.-G. Zheng, "Synthesis and application of stable copper oxide nanoparticle suspensions for nanoparticulate film fabrication," Journal of the American Ceramic Society, vol. 90, no. 1, pp. 107-110, 2007.

[21] J. Zhu, D. Li, H. Chen, X. Yang, L. Lu, and X. Wang, "Highly dispersed $\mathrm{CuO}$ nanoparticles prepared by a novel quickprecipitation method," Materials Letters, vol. 58, no. 26, pp. 3324-3327, 2004.

[22] A. S. Lanje, S. J. Sharma, R. B. Pode, and R. S. Ningthoujam, "Synthesis and optical characterization of copper oxide nanoparticles," Advances in Applied Science Research, vol. 1, no. 2, pp. 36-40, 2010.

[23] E. Lester, P. Blood, J. Denyer, D. Giddings, B. Azzopardi, and M. Poliakoff, "Reaction engineering: The supercritical water hydrothermal synthesis of nano-particles," Journal of Supercritical Fluids, vol. 37, no. 2, pp. 209-214, 2006.

[24] A. Cabañas, J. Li, P. Blood et al., "Synthesis of nanoparticulate yttrium aluminum garnet in supercritical water-ethanol mixtures," Journal of Supercritical Fluids, vol. 40, no. 2, pp. 284-292, 2007.

[25] C. J. Tighe, R. Q. Cabrera, R. I. Gruar, and J. A. Darr, "Scale up production of nanoparticles: continuous supercritical water synthesis of Ce-Zn oxides," Industrial and Engineering Chemistry Research, vol. 52, no. 16, pp. 5522-5528, 2013.

[26] W. Sigmund, J. Yuh, H. Park et al., "Processing and structure relationships in electrospinning of ceramic fiber systems," Journal of the American Ceramic Society, vol. 89, no. 2, pp. 395407, 2006

[27] A. Khalil, R. Hashaikeh, and M. Jouiad, "Synthesis and morphology analysis of electrospun copper nanowires," Journal of Materials Science, vol. 49, pp. 3052-3065, 2014.

[28] J. M. Deitzel, J. Kleinmeyer, D. Harris, and N. C. Beck Tan, “The effect of processing variables on the morphology of electrospun nanofibers and textiles," Polymer, vol. 42, no. 1, pp. 261-272, 2001.

[29] S. Megelski, J. S. Stephens, D. Bruce Chase, and J. F. Rabolt, "Micro- and nanostructured surface morphology on electrospun polymer fibers," Macromolecules, vol. 35, no. 22, pp. 84568466, 2002.

[30] M. M. Hohman, M. Shin, G. Rutledge, and M. P. Brenner, "Electrospinning and electrically forced jets. I. Stability theory," Physics of Fluids, vol. 13, no. 8, pp. 2201-2220, 2001.

[31] “JCPDS card no. 05-0661," 1986.

[32] R. Greenwood and K. Kendall, "Selection of suitable dispersants for aqueous suspensions of zirconia and titania powders using acoustophoresis," Journal of the European Ceramic Society, vol. 19, no. 4, pp. 479-488, 1999.

[33] M. M. Demir, M. A. Gulgun, Y. Z. Menceloglu et al., "Palladium nanoparticles by electrospinning from poly(acrylonitrile-coacrylic acid)-PdCl2 solutions: relations between preparation conditions, particle size, and catalytic activity," Macromolecules, vol. 37, no. 5, pp. 1787-1792, 2004.

[34] H. Dou and J. H. He, "Nanoparticles fabricated by the bubble electrospinning," Thermal Science, vol. 16, no. 5, pp. 1562-1563, 2012. 

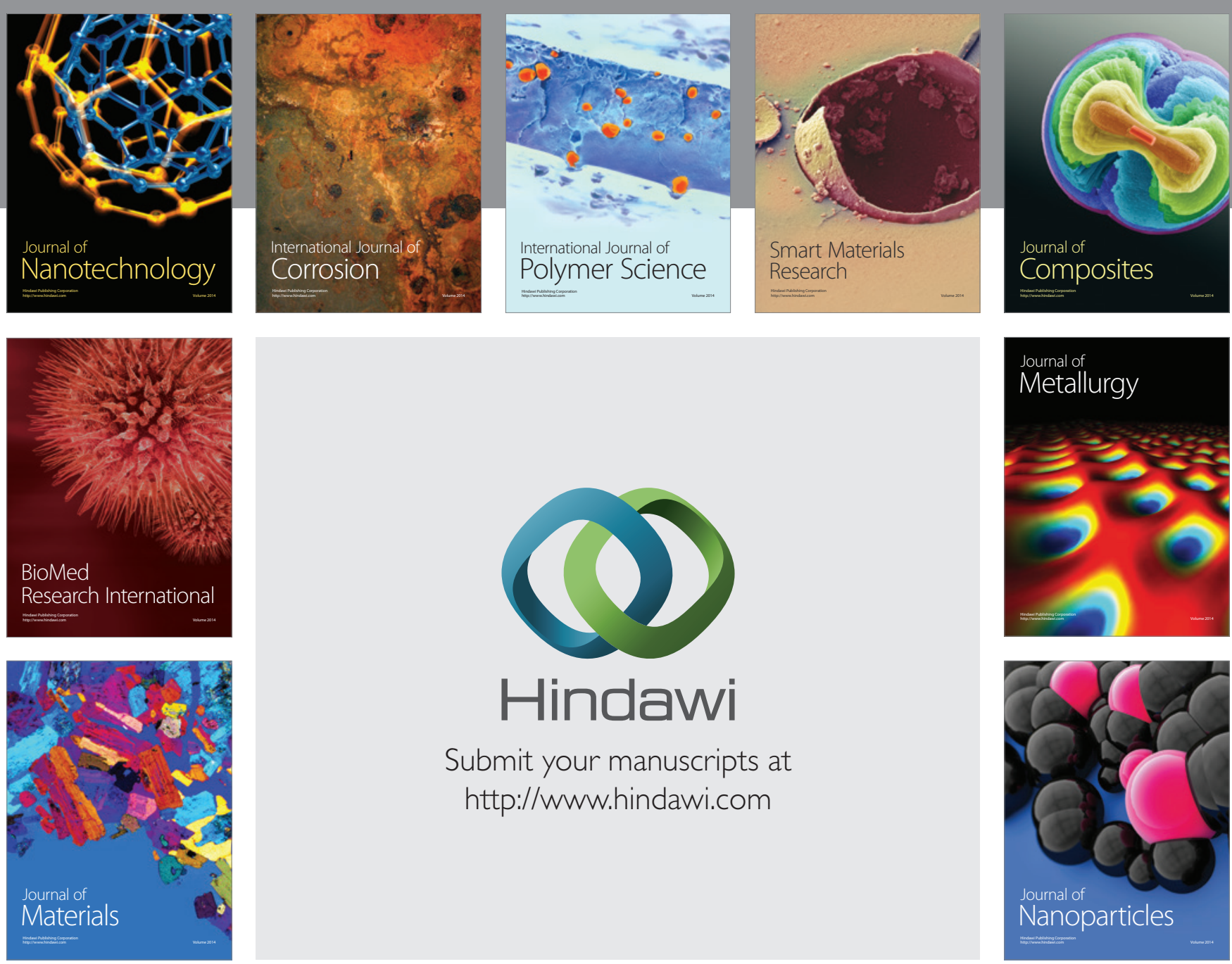

Submit your manuscripts at http://www.hindawi.com
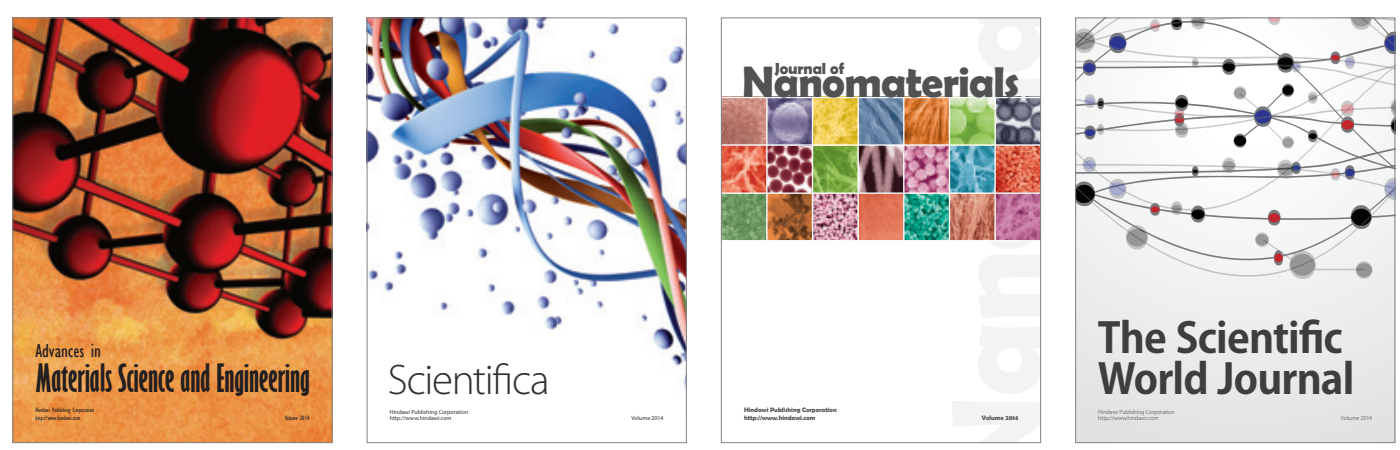

\section{The Scientific World Journal}
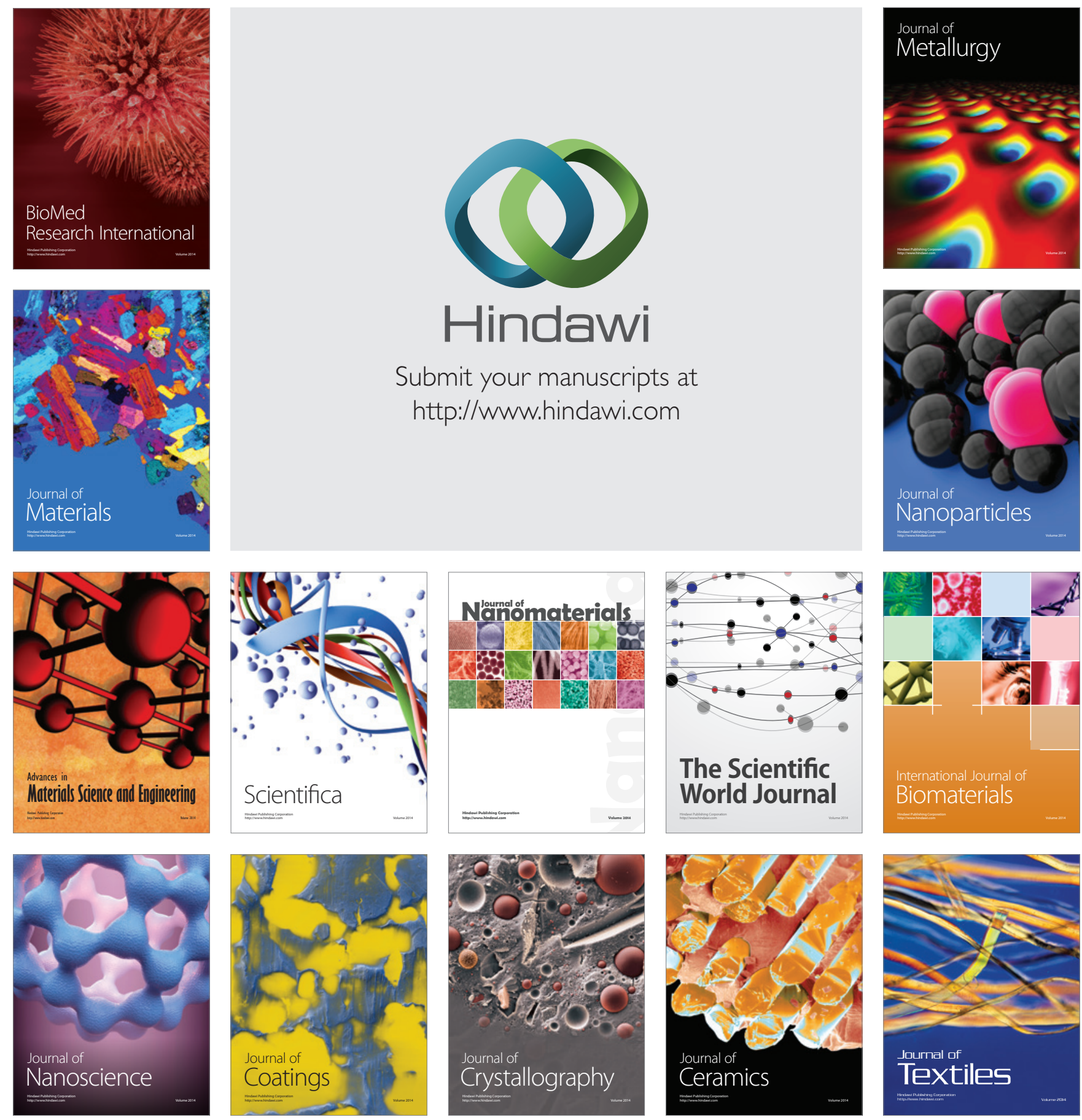